\title{
Electron Ionization-Tandem Mass Spectrometry of Glycosphingolipids. I: The Identification of Compound-specific Sequence Ions in the Collision-induced Dissociation Spectra of the Immonium Ions of Two Isomeric Hexaglycosylceramides
}

\author{
J. M. Curtis and P. J. Derrick \\ Warwick Institute for Mass Spectrometry and Department of Chemistry, University of Warwick, Coventry \\ CV4 7AL, UK
}

\author{
J. Holgersson and B. E. Samuelsson \\ Department of Medical Biochemistry, University of Göteborg, Göteborg, Sweden
}

\author{
M. E. Breimer \\ Department of Surgery, Sahlgren's Hospital, University of Göteborg, Göteborg, Sweden
}

\begin{abstract}
A permethylated-reduced hexaglycosylceramide in a complex glycolipid mixture isolated from a unique human tissue has been identified by using tandem mass spectrometry (MS/MS). The mass spectrum of this glycolipid mixture, obtained by using in-beam electron ionization, is very complex, and fragment ions derived from the hexaglycosylceramide cannot be distinguished from other ions. Tandem mass spectrometry using a four-sector mass spectrometer gave the mass spectrum of the immonium ion of the permethylated-reduced hexaglycosylceramide $(\mathrm{m} / \mathrm{z}$ 1645.8), which is characteristic of its structure. Comparison of this MS/MS spectrum with those of two similarly derivatized blood group hexaglycosylceramide isomers permitted identification of the unknown glycolipid structure. (J Am Soc Mass Spectrom 1992, 3, 353-359)
\end{abstract}

\begin{abstract}
$\mathrm{C}$ ell membrane glycolipids occur in biological systems as complex mixtures whose composiis specific to species, individuals, tissues, and cells $[1,2]$. Their particular biomedical importance is due to their activity as determinants within the major blood group systems (ABO, Lewis, X, I, and P) $[1,3]$. Their biological properties as transplantation antigens, tumor-associated antigens, and receptors for micrubes [1-5] make their structural characterization important. The complex nature of glycolipid fractions necessitates the use of analytical techniques that combine high sensitivity and structural specificity with the ability to analyze components from a mixture.

Recently, a number of chromatographic techniques have been used in conjunction with mass spectrome-
\end{abstract}

Address reprint requests to J. M. Curtis, Department of Chemistry University of Warwick, Coventry CV4 7AL, UK. try to tackle such problems, including gas chromatography mass spectrometry (GC/MS) [6], liquid chromatography mass spectrometry (LC/MS) [7], thin-layer chromatography mass spectrometry (TLC/MS) [8, 9], and supercritical fluid chromatography mass spectrometry (SFC/MS) [10]. The introduction of the permethylated and permethylated-reduced derivatives of glycolipids for electron ionization (EI/MS) analysis of pure glycolipids [11-13] contributed significantly to the structural analysis of small amounts of glycolipids. This was further developed into the analysis of sample glycolipid mixtures [13-15] by taking advantage of the difference in the temperatures at which glycolipids of different molecular weights evaporate. In this experiment, the cuvette containing the glycolipid sample is heated while spectra are recorded continuously as the glycolipid species evaporate. By placing the cuvette close to the ionizing electron beam, it has been possible to obtain spectra 
from glycolipids with up to 12 sugars as pure compounds $[16\rceil$ and with up to 11 sugars in a mixture [15].

Underivatized glycolipids have also been studied by "soft" ionization techniques, especially fast atom bombardment (FAB/MS) [12-19]. However, FAB/MS has serious disadvantages when applied to mixtures of glycosphingolipids (GSI.s), which exhibit heterngeneity in both saccharide and ceramide moieties. First, because all components are ionized more or less simultaneously, FAB/MS does not give the advantage of partial separation by distillation. The inherent difficulty with FAB/MS of glycolipids in distinguishing between molecule ions and fragment ions is thus not facilitated and this becomes increasingly difficult with increasing molecular size. It may be partially overcome by the use of FAB ionization in conjunction with tandem mass spectrometry (FAB/MS/MS) [17], but this approach may not always be feasible. Another disadvantage of $F A B$ ionization applied to mixtures is the effect of competitive ionization [20], whereby one or more components dominate the spectrum so that other possibly major components appear as only weak peaks in the spectrum, which may be overlooked on interpretation. However, a major advantage of FAB/MS is that prior derivatization of the glycolipids is not required.

Another approach to glycolipid analysis was recently described [21] which employed an electrospray ionization (ESI) source combined with a triplequadrupole mass spectrometer. The doubly charged molecular ions of two gangliosides $\left(M_{r} \approx 2000\right)$ in positive ion mode showed considerable heterogeneity, covering $\sim 80$ mass-to-charge units. Signifcantly, in negative ion mode the spectrum around the doubly charged molecular ion region was greatly simplified. The combination of ESI with high performance MS/MS may prove to be a powerful tool for glycolipid analysis in the future.

A major advance in the study of large biomolecules has been the development of "high performance" MS/MS by combining two high resolution doublefocusing mass spectrometers [22-24]. Unit mass parent ion resolution is essential for collision-induced dissociation experiments on samples of biological origin because these often contain variants of similar mass. In addition, isotopic contributions become increasingly significant at higher mass and these complicate the daughter ion spectrum. Daughter ion mass accuracies of better than $\pm 0.3 \mathrm{u}$ are required to unambiguously assign peaks. The necessity for high energy collisions has been widely discussed [25]. These criteria, which are essential for structural analysis, are all achieved by current commercial four-sector instruments that have yielded MS/MS spectra on molecules of up to $\sim 2500 \mathbf{u}$.

In a recent publication [15], we have highlighted the potential use of a four-sector instrument to improve the level of structural information obtainable from the high mass compounds using the distillationEI method described above. It was found that as the complexity of the mixture increases, so the interpretation of the resulting spectra becomes increasingly ambiguous, for three main reasons. First, in a complex mixture immonium ions from a given GSL are not easily discriminated from fragment ions (sequence ions) from other GSLs. Second, the origins of fragment ions from different GSLs are not easily established. Third, the number of possible isomeric structures giving rise to isobaric immonium ions increases, especially with increasing mass. For a given fatty acid-containing immonium ion, these isomeric structures can arise due to different linkages within the carbohydrate chain, as well as different carbohydrate sequences.

The present work describes the analysis of two blood group hexaglycosylceramide isomers by EI/MS/MS and the application of this technique to the successful identification of a hexaglycosylceramide in a glycolipid mixture isolated from a unique human tissue sample.

\section{Experimental}

\section{Sample Preparation}

Reference glycolipids. Blood group Le $\mathrm{b}^{\mathrm{b}}$ and $\mathrm{B}$ hexaglycosylceramides were isolated from human small intestine and pancreas, respectively [26]. Their structures were established by mass spectrometry, proton nuclear magnetic resonance spectroscopy and degradation analysis [26]. The EI mass spectra of the permethylated and the permethylated-reduced blood group B compound are described in Breimer [26], and the spectra of the corresponding $\mathrm{Le}^{\mathrm{b}}$ glycolipid isolated from human plasma in Falk et al. [27].

Human liver glycolipid fraction. The liver from a blood group $A_{1} \operatorname{Le}(a-b+)$ human individual was transplanted into a blood group $O$ recipient. Because of incompatible organ size, part of the liver was resected and analyzed. A total nonacid glycolipid fraction was prepared as described [28], and by high performance liquid chromatography a blood group glycolipid fraction migrating in the $6 / 7$ sugar region on the thin-layer plate was isolated. A detailed structural investigation of the liver glycolipids (to be published elsewhere) revealed for the sample analyzed in this article a blood group A difucosyl heptaglycosylceramide as major component, a Le ${ }^{b}$ hexaglycosylceramide, and traces of a blood group A monofucosylhexaglycosylceramide.

Derivatizations. The reference glycolipids were permethylated by using the method of Hakomori [29] and the liver sample by the method of Ciucanu and Kerek [30] and Larson et al. [31]. The permethylated 
glycolipids were reduced by $\mathrm{LiAlH}_{4}$ in diethyl ether as described [32].

\section{Mass Spectrometry}

The mass spectrum shown in Figure 1 was recorded at the University of Göteborg by using a VG Instruments Analytical ZAB-2F spectrometer (Manchester, UK), as described previously [13-15].

All other spectra shown were obtained by using a Kratos Analytical Concept II $\mathrm{HH}$ four-sector mass spectrometer (Manchester, UK) at the University of Warwick. In this method 1-10 $\mu \mathrm{g}$ of the permethylated-reduced GSL samples were loaded onto a ceramic probe-tip having adjustable length and orientation. The tip was placed 1-2 mm from the electron beam and heated only via contact with the source block. The source block temperature was manually ramped between 200 and $320{ }^{\circ} \mathrm{C}$ until appropriate conditions were found. Under these conditions the reference glycolipids gave signals lasting up to $5 \mathrm{~min}$. The human liver fraction gave less stable signals lasting only $\sim 2 \mathrm{~min}$. The accelerating voltage was $8 \mathrm{kV}$, the electron energy $100 \mathrm{eV}$, and the trap stabilization current $500 \mu \mathrm{A}$. The instrument was operated with an MS-I resolution of 2000 , and MS-II resolution of 1000 . All tandem mass spectra were obtained with a collision energy of $6 \mathrm{keV}$ and a $60 \%$ beam attenuation using $\mathrm{He}$ collision gas. $A$ scan specd of $10 \mathrm{~s} /$ decade was used for the mass spectra shown in Figure 1. All tandem mass spectra were recorded as "raw data," using a scan speed of $30 \mathrm{~s} /$ decade and averaging three to five scans.

\section{Results and Discussion}

The mass spectra of the permethylated-reduced blood group $B$ and $\mathrm{Le}^{\mathrm{b}}$ hexaglycosylceramides are shown in Figure 2. As described previously [11], such spectra are dominated by immonium ions which arise by loss of the alkyl group associated with the long chain base with retention of the alkyl group from the acyl moiety. These immonium ions are evident $\sim m / z$ 1600-1650 (Figure $2 a$ and $b$ ). The series of immonium ions is due to variation in the chain length and hydroxylation of the fatty acid. Of particular interest are the immonium ions at $m / z 1645.8$, whose structures are shown in Scheme I. This example illustrates how, as the molecular mass of the glycolipid increases, the interpretation of the resulting spectra becomes increasingly ambiguous. Thus, with increasing sugarchain length the number of possible structures for each series of immonium ions which retain the alkyl group from the acyl moiety (fatty acid), increases. For example, an exchange of a hexose for a deoxyhexose residue means loss of 30 mass units, which is the same as the loss obtained by substitution of a hydroxy fatty acid by a non-hydroxy acid in the lipophilis part of the GSL [11, 12]. Thus, a given immonium ion could correspond to either a structure containing a hexose plus non-hydroxy fatty acid, or a structure containing a deoxyhexose plus hydroxy fatty acid. The two immonium ions shown in Scheme I differ in exactly this way, and so give rise to isobaric immonium ions at $m / z$ 1645.8. In the case of the purified glycolipids, the mass spectra show series of sequence ions which readily distinguish the two structures (Figure 2 and Scheme I). For example, in the blood group B GSL spectrum, a fragment ion appears at $\mathrm{m} / \mathrm{z}$ 828.3, which is due to the tetrasaccharide Hex-OHex(-O-Fuc)-O-HexNAc. The corresponding ion in the blood group Le $e^{b}$ GSL spectrum appears at $m / z 798.3$, owing to the tetrasaccharide fragment Hex(-O-Fuc)HexNAc-(O-Fuc), i.e., with one hexose replaced by deoxyhexose (fucose) resulting in a shift of $30 \mathrm{u}$. However, in a mixture the glycolipids give rise to a vastly more complex mass spectrum, as illustrated by the spectrum of the human liver glycolipid mixture (Figure 1) in which any sequence ions are indistinguishable from, or buried beneath, ions arising from other components of the mixture (Samuelsson et al., to be published). Thus, the immonium ion at $\mathrm{m} / \mathrm{z}$ 1645 could be assigned to either a blood group B or a blood group Le $\mathrm{L}^{\mathrm{b}}$ hexaglycosylceramide structure.

In contrast to this situation, the tandem mass spectrum contains only ions which are fragments of a preselected parent ion, provided that the mass resolution of MS-I is sufficient to resolve the parent ion from its nearest neighbor. Therefore, it was hoped that by recording the tandem mass spectra of the isobaric immonium ions at $m / z 1645.8$ of the GSLs of blood groups $B$ and $\mathrm{Le}^{\mathrm{b}}$, it would be possible unambiguously to identify their sequence. This technique could then be applied to complex glycolipid mixtures where sequence ions are often difficult to identify. Figure $3 a$ and $b$ shows the resulting tandem mass spectra, recorded using an MSI resolution of 2000 . The tandem mass spectra of the immonium ions of the two hexaglycosylceramides clearly identify the structures as being those of the blood group $\mathrm{B}$ and $\mathrm{Le}^{\mathrm{b}}$ compounds. Structurally significant ions at $m / z$ 157, 189, $424,798,1002,1176,1206,1485,1600$, and 1613 are present in the spectrum of the $\mathrm{Le}^{\mathrm{b}}$ compound (Figure $3 \mathrm{~b})$. In the tandem mass spectrum of the immonium ion of the blood group B compound (Figure 3a), peaks are seen at $m / z$ 394, 596, 828, 1048, 1206, and 1455 corresponding to the sequence shown in Scheme Ia. An ion of $m / z 1206$ is also present in the Le ${ }^{b}$ spectrum, and is due to the loss of the hexasaccharide (Scheme $\mathrm{Ib}$ ). The equivalent loss for the blood group B immonium ion results in a fragment ion of $\mathrm{m} / \mathrm{z}$ 1236 (30 mass units higher due to one deoxyhexose being replaced by hexose). The fragment ion at $m / z$ 1206 for the blood group B immonium ion may be due to loss of both the terminal hexose and fucose (Scheme Ia). This idea is supported by the presence of weaker ions at $\mathrm{m} / \mathrm{z} 1222$ and 1236 , representing retention of the glycosidic linkages. 

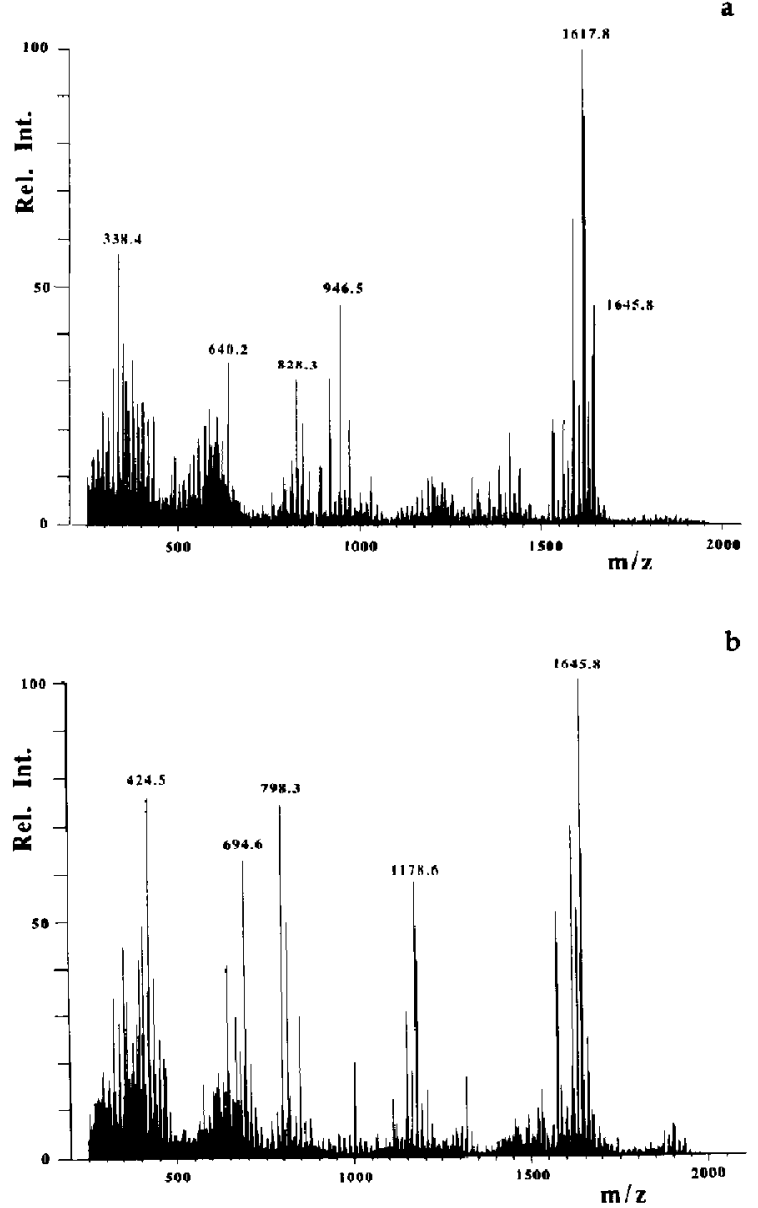

Figure 1. "In beam" EI mass spectra of the permethylatedreduced hexaglycosylceramides of (a) blood group B, (b) blood group Le $\mathrm{e}^{\mathrm{t}}$.
The EI/MS/MS technique was then applied to a mixture of glycolipids isolated from a liver of a blood group A, Le $(a-b+)$ human individual. In this way it was hoped that sequence data could be obtained for a single fatty acid-containing immonium ion present in the complex spectrum shown in Figure 2. The resulting tandem mass spectrum of $m / z 1645.8$ (Figure 3c) shows fragment ions at $m / z 157,189,424$, $798,1002,1176,1206,1485,1600$, and 1613, corresponding to the sequence of the $\mathrm{Le}^{\mathrm{b}}-6$ compound, (compare Figure 3b). This clearly establishes the presence of the Le $^{\mathrm{b}}-6$ compound in the liver glycolipid mixture, despite the rather poor signal-to-noise level shown in Figure 3c. The latter is in part due to the transient nature of the parent ion signal recorded using the distillation-EI technique on such a mixture.

This work illustrates the utility of four-sector mass spectrometry in the direct structural analysis of molecules in the low kilodalton range from highly complex mixtures of biological origin. This was achieved without the use of on-line chromatography.

The major drawback of the method lies in the difficulty in achieving sufficiently intense parent ions required to obtain acceptable tandem mass spectra, from trace amounts of material. For example, the tandem mass spectrum shown in Figure $3 \mathrm{~b}$ results from a parent ion signal in the range $300-800 \mathrm{mV}$ at a gain of $10^{6}$, and lasting long enough for one to two scans. The disappointing signal-to-noise level seen in the tandem mass spectrum obtained from the liver extract illustrates the need for greater sensitivity. However, it may be that a $1-10-\mu \mathrm{g}$ sample represents a mixture of several compounds and one immonium

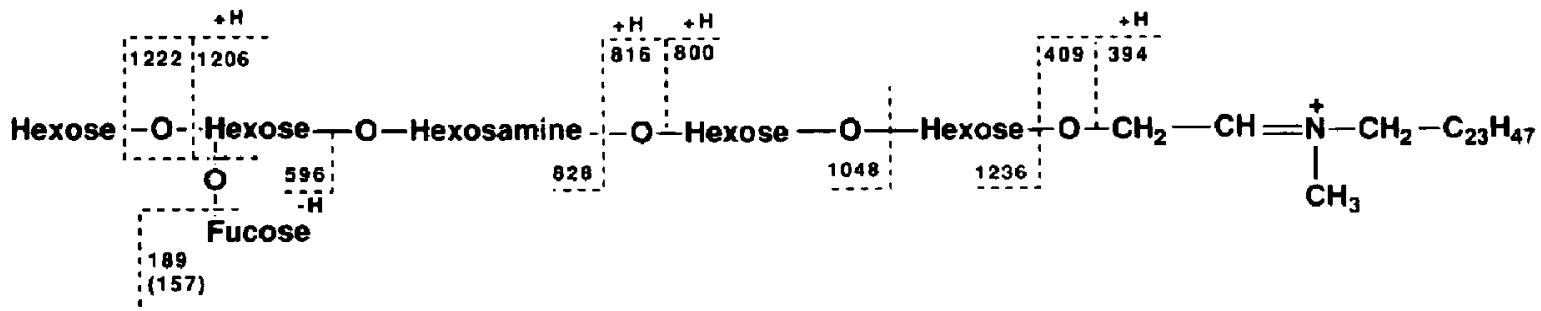

a

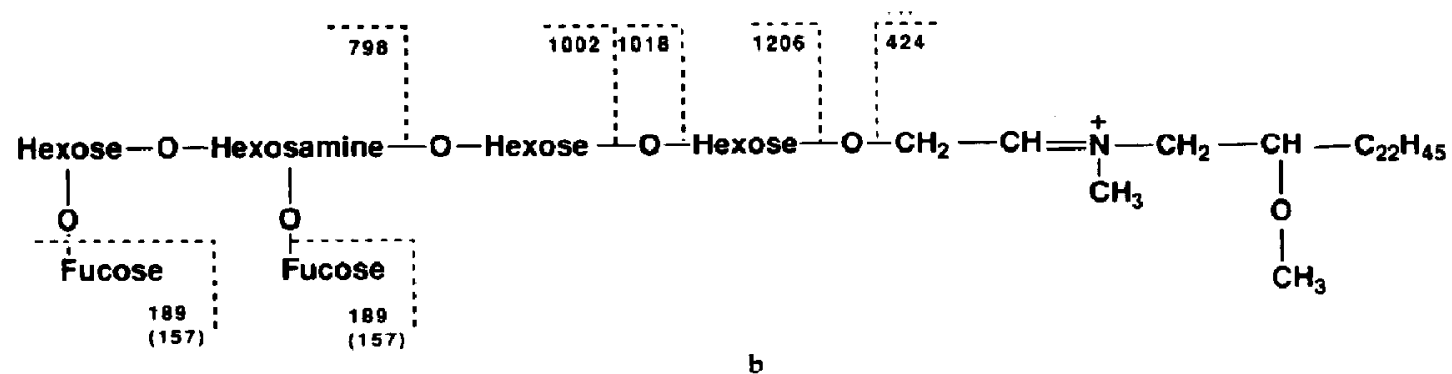

Scheme I. Immonium ions of the permethylated-reduced hexaglycosylceramides at $\mathrm{m} / \mathrm{z} 1645.8$ for (a) blood group B, (b) blood group Le, showing modes of fragmentation seen in MS and MS/MS spectra. 

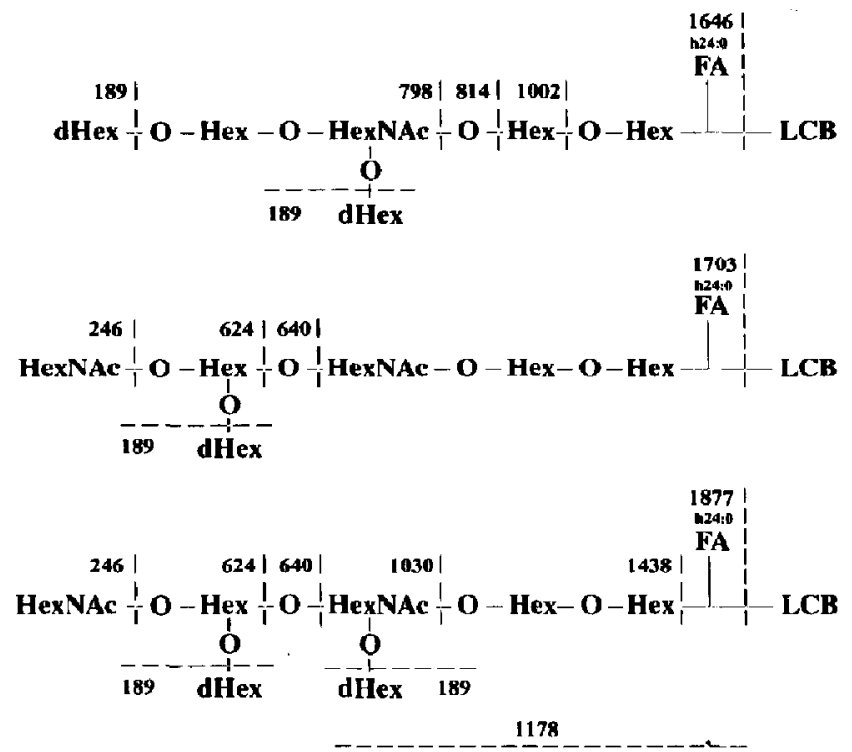

Rel. Int.

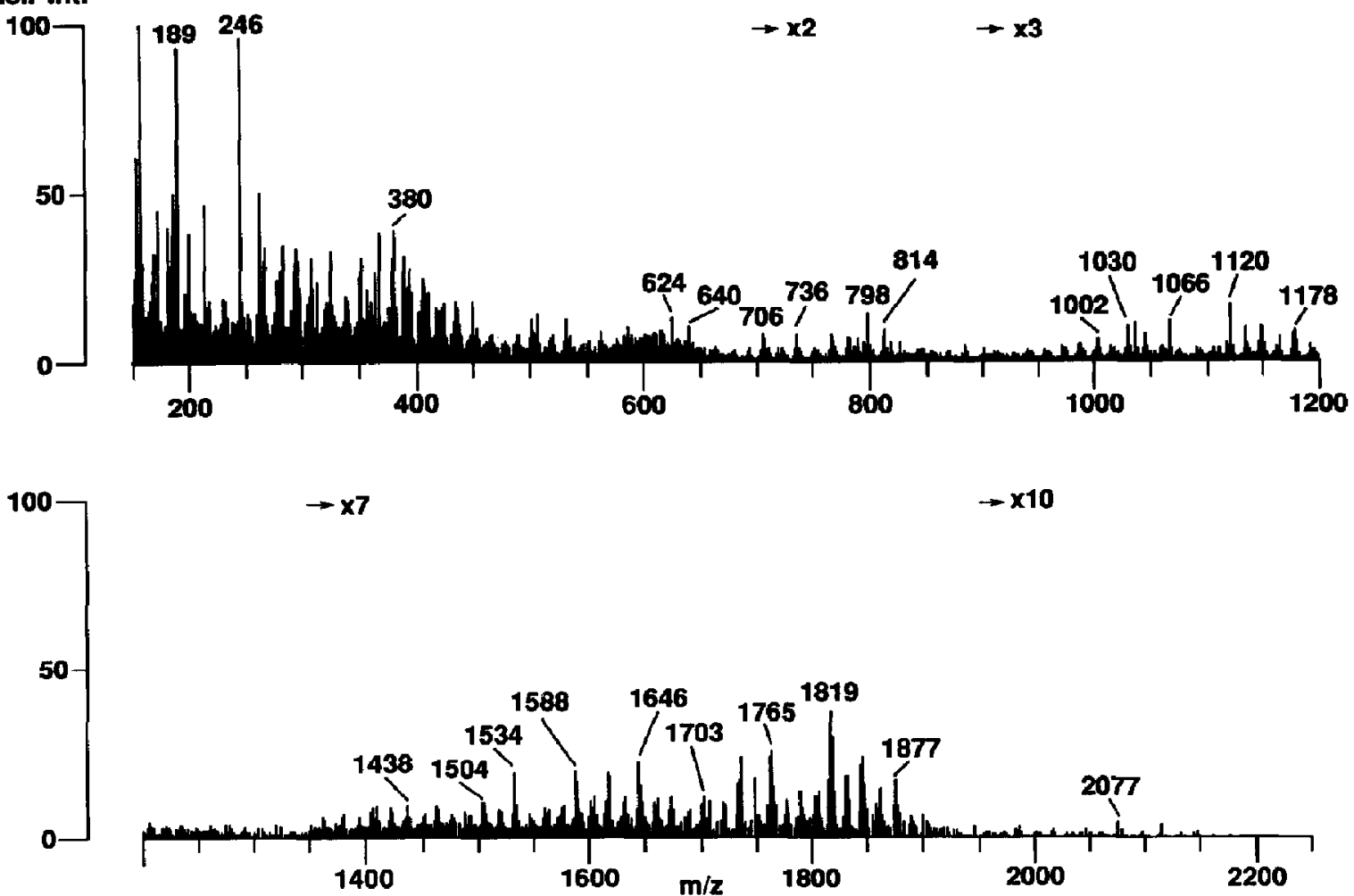

Figure 2. "In beam" EI mass spectrum of permethylated-reduced human liver glycolipid fraction obtained by using a VG Instruments $\mathrm{ZAB}-2 \mathrm{~F}$. The sample was introduced "in beam" at $300^{\circ} \mathrm{C}$ and the source temperature was ramped at $6^{\circ} \mathrm{C}$ per minute. Mass spectra were recorded continuously. The reproduced spectrum is 15 out of 22. Trap current was $200 \mu \mathrm{A}$ and electron energy $52 \mathrm{eV}$. Simplified formulas are shown above the spectrum for interpretation. 


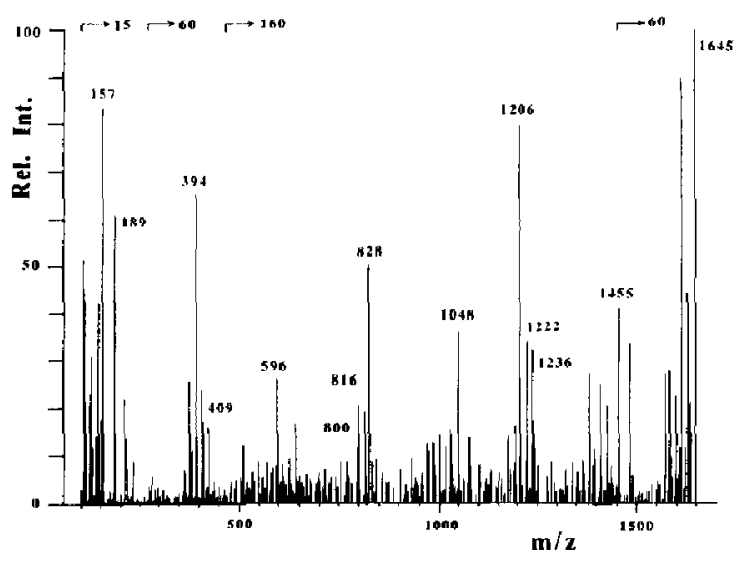

a
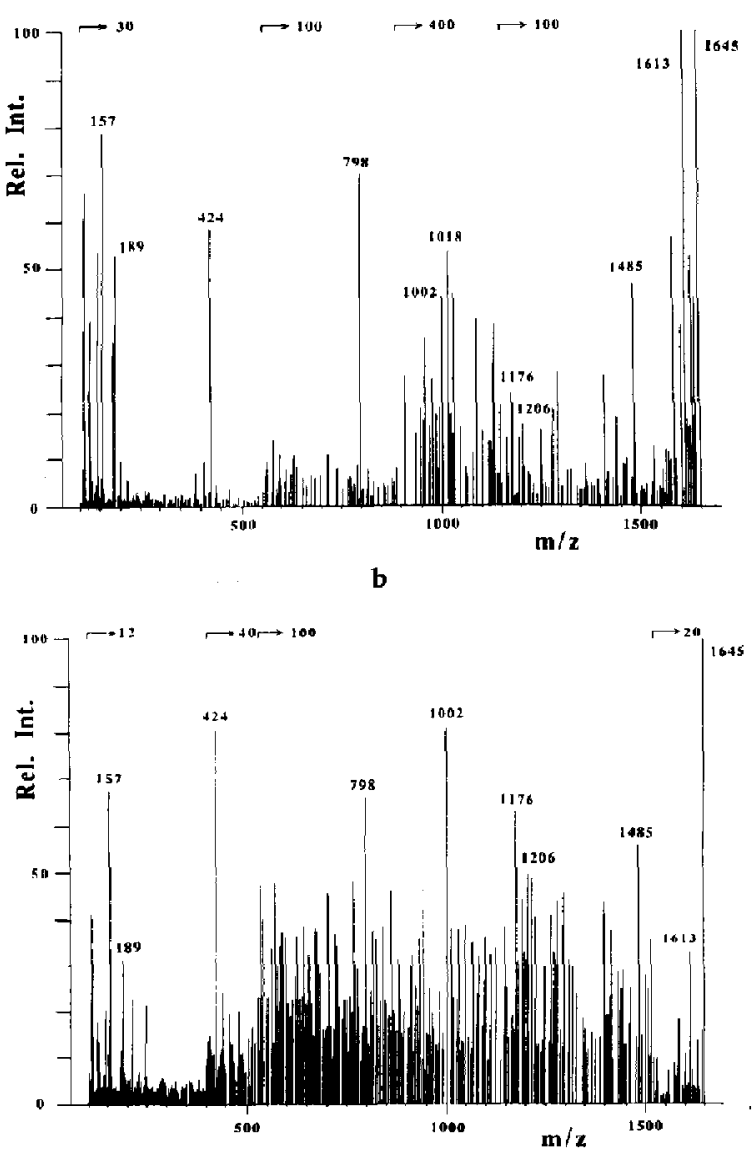

c

Figure 3. MS/MS spectra of the immonium ions of $m / z 1645.8$ of the permethylated-reduced hexaglycosylceramides of (a) blood group B, (b) blood group Le ${ }^{\mathbf{b}}$, (c) human liver extract. Spectra were recorded at a collision energy of $6 \mathrm{keV}$ using $\mathrm{He}$ collision gas. ion represents one species out of several (16-26 carbon fatty acids, hydroxy, or non-hydroxy). In such a perspective the sensitivity is reasonable. The most promising development allowing such an increase in sensitivity is the recent use $[33,34]$ of the simultaneous detection of ions over a given mass range using electrooptical multichannel array detectors. Such detection system can result in up to one hundredfold increase in sensitivity [33] over conventional point detectors, such as the electron multiplier used in the present work. It is hoped for the future that by combining the benefits of array detection with some refinements to the ionization technique, the identifcation of GSI ss by MS/MS could become a well-estahlished procedure. This would have major benefits in the area of organ transplantation, where donor organ blood group phenotyping on small tissue samples might be a prerequisite for carbohydrate-based blood group compatibility between donor organ and recipient, thus resulting in a good graft survival.

\section{References}

1. Clausen, H.; Hakomori, S. Vox Sang. 1989, 56, 1.

2. Holgersson, J.; Clausen. H.; Hakomori, S.; Samuelsson, B. E.; Breimer, M. B. J. Biol. Chem. 1990, 256, 20790.

3. Watkins, W. M. Adv. Hum. Genet. 1980, 10, 1.

4. Hakomori, S. Chem. Phys. Lipids 1986, 42, 209.

5. Karlsson, K.-A. Annu. Rev. Biochem. 1989, 58, 309.

6. Karlsson, H.; Carlstedt, I.; Hansson, G. C. FEBS Lett. 1987, 226, 23.

7. Evans, J. E.; McCluer, R. H. Biomed. Mass Spectrom. 1987, 14, 149.

8. Kushi, Y.; Rokukawa, C.; Handa, S. Anal. Biochem. 1988, $175,167$.

9. Samuelsson, B. E.; Pimlott, W.; Karlsson, K.-A. Methods Enzymol. 1988, 193, 623-646.

10. Reinhold, V. N.; Sheeley, D. M.; Kuei, J.; Her, G.-R. Anal. Chem. 1988, 60, 2719.

11. Karlsson, K.-A. FEBS Lett. 1973, 32, 317.

12. Karlsson, K.-A.; Pascher, J.; Pimlott, W.; Samuelsson, B. E. Biamed. Mass Spectrom. 1974, 1, 79.

13. Breimer, M. E.; Hansson, G. C.; Karlsson, K.-A.; Leffler, H.; Pimlott, W.; Samuelsson, B. E. Biomed. Mass Spectrom. 1979, 6, 231.

14. Samuelsson, B. E. In Advances in Mass Spectrometry; Todd, J. F. J., Ed.; Wiley: Chichester, U.K., 1986; p 225.

15. Holgersson, J.; Pimlott, W.; Samuelsson, B. E.; Breimer, M. E. Rapid Commun. Mass Spectrom. 1989, 3, 400.

16. Breimer, M. E.; Hansson, G. C.; Karlsson, K.-A.; Leffler, H.; Pimlutt, W.; Samuelssun, B. E. FEBS Letl. 1981, 124, 299.

17. Domon, B.; Costello, C. E. Biochemistry 1988, 27, 1534.

18. Hemling, M. E.; Yu, R. K.; Sedgwick, D.; Rinehart, K. L. Jr. Biochemistry 1984, 23, 5706.

19. Levery, S. B.; Salyan, M. E. K.; Roberts, C. E.; Hakomori, S. In Proceedings of the 38th ASMS Conference on Mass Spectrometry and Allied Topics. Tucson, AZ, June 3-8, 1990; PP 1369-1370.

20. Biemann, K.; Martin, S. A. Mass Spectrom. Rev. 1987, 6, 1.

21. Huang, E. C.; Henion, J. D. In Proceedings of the 38th ASMS 
Conference on Mass Spectrometry and Allied Topics, Tucson, $\mathrm{AZ}$, June 3-8, 1990; pp 291-292.

22. McLafferty, F. W.; Todd, P. J.; McGilveray, C.; Baldwin, M. A. I. Am. Chem. Soc. 1980, 102, 3360.

23. Boyd, R. K.; Bott, P. A.; Harvan, D. J.; Hass, J. R. Int. J. Mass Spectrom. Ion Processes 1986, 69, 251.

24. Sato, K.; Asada, T.; Isihara, M.; Kunihiro, F.; Kammai, Y.; Kubota, E.; Costello, C. E.; Martin, S. A.; Scoble, H. A.; Biemann, K. Artal. Chem. 1988, 59, 1652.

25. Alexander, A. J.; Thibault, P.; Boyd, R. K.; Curtis, J. M.; Rinehart, K. L. Int. J. Mass Spectrom. Ion Processes 1990, 98, 107.

26. Breimer, M. E. Arch. Biockem. Biophys. 1984, 228, 71 .
27. Falk, K.-E.; Karlsson, K.-A.; Samuelsson, B. E. FEBS Lett. 1981, 124, 173.

28. Karlsson, K.-A. Methods Enzymol. 1987, 138, 212.

29. Hakomori, S. I. Biochem. 1964, 55, 205.

30. Ciucanu, I.; Kerek, F. Carbohyd. Res. 1987, 138, 99.

31. Larson, G.; Karlsson, H.; Hansson, G. C.; Pimlott, W. Carbohyd. Res. 1987, 161, 281.

32. Karlsson, K.-A, Biochemistry 1974, 13, 3643.

33. Cotrell, J. S.; Evans, S. Anal. Chem. 1987, 59, 1990.

34. Hill, J. A.; Biller, J. E.; Martin, S. A.; Biemann, K.; Yoshidome, K.; Sato, K. Int. J. Mass Spectrom. Ion Processes 1989, 92, 211. 